\title{
Reconstruction of longitudinal distributed incoherent sources
}

\author{
Joseph Rosen* and Amnon Yariv \\ California Institute of Technology, M/S 128-95, Pasadena, California 91125
}

Received June 6, 1996

\begin{abstract}
We describe measurement of the degree of coherence induced by a random light source distributed along the longitudinal $z$ axis. If this degree of coherence is measured only between all the in-plane pairs of points placed along the radial lines it is proportional to the Fourier transform of the source's three-dimensional intensity distribution as seen from the paraxial far zone. A reconstruction of the source shape from the measured degree of coherence is also demonstrated. (c) 1996 Optical Society of America
\end{abstract}

Imaging by a very long-baseline interferometer ${ }^{1}$ can be explained by the relation between the far-field degree of coherence and the intensity distribution of the imaged object as formulated by the Van CittertZernike theorem. Explicitly, it is manifested in this theorem that the two-point degree of coherence in the far field of a quasi-monochromatic, spatially incoherent light source is proportional to the Fourier transform of the source's planar intensity distribution. This longterm theory was updated recently because new results were obtained ${ }^{2,3}$ that indicate that the far-field degree of coherence is modified in a particular way if the source is distributed along the longitudinal axis.

In Refs. 2 and 3 we presented the two-point degree of coherence induced by a three-dimensional (3-D) quasimonochromatic incoherent source from the paraxial far zone. Two different schemes were proposed to measure this degree for two applications. In one scheme the degree of coherence degenerates into a twodimensional (2-D) function containing the information about the 3-D source in the same manner as appears in a coherently photographed hologram. The complex distribution of this degree of coherence is actually analogous to a Fourier hologram. Therefore, by illuminating a hologram with a transparency distribution of this degree of coherence in front of a spherical lens, we reconstructed the 3-D object in the vicinity of the lens's back focal plane. ${ }^{2}$ Inherently the 2-D hologram cannot contain all the 3-D information about the original object. Therefore this hologram cannot be transformed exactly back into the 3-D object, a problem that also exists in other conventional holograms.

To overcome this holographic ambiguity problem we proposed recording a 3-D degree of coherence and having a precise representation of the 3-D intensity distribution emitted from the source. ${ }^{3}$ The precise reconstruction is achievable because this degree of coherence is proportional to the 3-D Fourier transform of the intensity distribution emitted from the 3-D source and observed form the far paraxial zone.

In this Letter we present an experimental demonstration of the degree of coherence induced by a longitudinal distributed incoherent source. This degree of coherence is obtained in the form of a complex visibility function of interference gratings in a Youngexperiment configuration. ${ }^{4}$ Although a 3-D system is considered in the theory, we simplify the experimental setup by measuring a complex visibility along only one transverse coordinate, say, $x$, understanding that the extension to the additional transverse dimension $y$ is straightforward. The reconstruction of the source's distribution from the measured complex visibility is described below.

A typical configuration is shown in Fig. 1. A 3-D light source $I_{s}\left(\mathbf{r}_{s}\right)$ in the coordinates $\left[\mathbf{r}_{s}=\left(x_{s}, y_{s}, z_{s}\right)\right.$ ] illuminates, among others, two points on the $x$ $y$ plane located far from the source. We assume that the source is a collection of incoherent quasimonochromatic point sources of spherical waves. Starting our analysis from a well-known expression ${ }^{5}$ generalized in a straightforward manner for 3-D sources, the mutual intensity between any two points on the $x-y$ plane with coordinates $\left(x_{i}, y_{i}\right), i=1,2$, is

$$
J\left(x_{1}, y_{1}, x_{2}, y_{2}\right)=C \int I_{s}\left(\mathbf{r}_{s}\right) \exp \left[-j k\left(R_{1}-R_{2}\right)\right] \mathrm{d}^{3} r_{s},
$$

where $k=2 \pi / \lambda, \lambda$ is the average wavelength, $R_{i}$ is the distance of a point source to the $i$ th observation point, $C$ is a constant, and the integration is done over a volume instead of over a plane as in Ref. 5. When simple geometrical arguments are employed to approximate the distance $\left(R_{1}-R_{2}\right)$, the mutual intensity becomes ${ }^{2}$

$$
\begin{aligned}
& J\left(x_{1}, y_{1}, x_{2}, y_{2}\right)=C \exp \left[\frac{j k(\hat{x} \Delta x+\hat{y} \Delta y)}{R}\right] \int I_{s}\left(\mathbf{r}_{s}\right) \\
& \quad \times \exp \left\{-j k\left[\frac{x_{s} \Delta x+y_{s} \Delta y}{R}+\frac{z_{s}(\hat{x} \Delta x+\hat{y} \Delta y)}{R^{2}}\right]\right\} \mathrm{d}^{3} r_{s},
\end{aligned}
$$

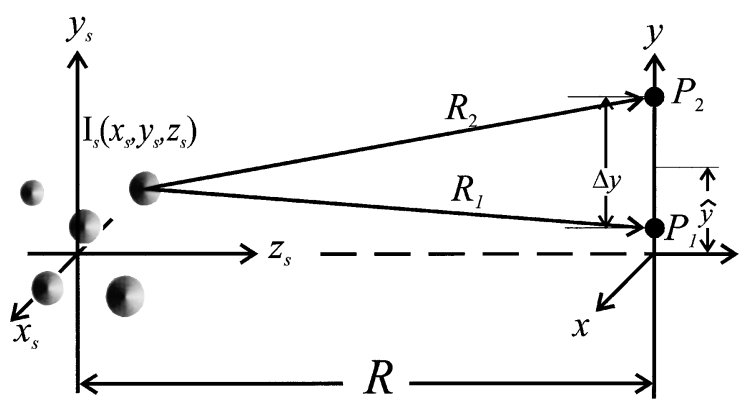

Fig. 1. Schematic illustration for calculation of the degree of coherence between $\mathrm{P}_{1}$ and $\mathrm{P}_{2}$.

(c) 1996 Optical Society of America 
where $\hat{x}=\left(x_{1}+x_{2}\right) / 2, \hat{y}=\left(y_{1}+y_{2}\right) / 2, \Delta x=x_{1}-x_{2}$, and $\Delta y=y_{1}-y_{2}$.

At first glance we note a few properties of this mutual intensity. First, it contains information about the depth dimension of the source through the variable $z_{s}$ in the exponent. Second, the modules of $J$, in general, are space variant because of the dependence on $\hat{x}$ and $\hat{y}$. Finally, although $J$ is space variant, the intensity distribution is uniform over the observation plane as in the case of a planar incoherent source. ${ }^{6}$ The far-field intensity distribution is given by

$$
I(x, y)=J(\Delta x=0, \Delta y=0)=C \int I_{s}\left(\mathbf{r}_{s}\right) \mathrm{d}^{3} r_{s} \equiv I_{0}
$$

We conclude that, in general, the modules of the mutual intensity induced by a 3-D incoherent source are space variant in a special way, depending on the product of the center of gravity of the measurement points $(\hat{x}, \hat{y})$ and the distance between them $(\Delta x, \Delta y)$; i.e.,

$$
\left|J\left(x_{1}, y_{1}, x_{2}, y_{2}\right)\right|=|J(\Delta x, \Delta y, \hat{x} \Delta x, \hat{y} \Delta y)| .
$$

By considering specific points on the $x-y$ plane, we reduce the four-dimensional mutual intensity given by Eq. (2) to a 3-D function. The desired points are all the pairs situated simultaneously on a radial line emanating from the origin. Formally, this restriction satisfies the condition $x_{1} y_{2}=x_{2} y_{1}$. If we use the relation $\hat{r} \Delta r=\hat{x} \Delta x+\hat{y} \Delta y$, the mutual intensity appears now in the general form of $J\left(x_{1}, y_{1}, x_{2}, y_{2}\right)=J(\Delta x, \Delta y, \hat{r} \Delta r)$, where $\hat{r}=\sqrt{\hat{x}^{2}+\hat{y}^{2}}$ and $\Delta r=\sqrt{\Delta x^{2}+\Delta y^{2}}$. Measuring the degree of coherence along these special points enables us to reconstruct (by a 3-D inverse Fourier transform) the 3-D intensity distribution of the source as seen from the observation plane. To see that, let us change the variable $\hat{r}$ to $q=\hat{r} \Delta r / \Delta r_{\min }$. Substituting the new variable into Eq. (2) and normalizing $J$ yield the following complex degree of coherence:

$$
\begin{aligned}
\mu(\Delta x, \Delta y, q) \equiv & \frac{J(\Delta x, \Delta y, \hat{r} \Delta r)}{I(x, y)} \\
= & C_{0} \int I_{s}\left(\mathbf{r}_{s}\right) \exp \left[\frac { - j 2 \pi } { \lambda } \left(\frac{x_{s} \Delta x+y_{s} \Delta y}{R}\right.\right. \\
& \left.\left.+\frac{z_{s} q \Delta r_{\min }}{R^{2}}\right)\right] \mathrm{d}^{3} r_{s}
\end{aligned}
$$

where $C_{0}=I_{0}^{-1} \exp \left(j k q \Delta r_{\min } / R\right)$. Equation (4) indicates that the degree of coherence along the coordinates $(\Delta x, \Delta y, q)$ is a 3-D Fourier transform (with scaling factors) of the source's 3-D intensity distribution, as seen from the far paraxial zone.

The experimental system is shown in Fig. 2. As mentioned above, we have reduced the system's dimensions to one transverse coordinate only for simplicity, realizing that the new physics (i.e., the relation between the far-field coherence and the source's depth distribution) is demonstrated as well in the reduced system. For this system Eq. (4) naturally becomes

$$
\begin{aligned}
\mu(\Delta x, p)= & I_{0}{ }^{-1} \exp \left(j K p \Delta k_{\min } / R\right) \\
& \times \iint I_{s}\left(x_{s}, z_{s}\right) \exp \left[\frac { - j 2 \pi } { \lambda } \left(\frac{x_{s} \Delta x}{R}\right.\right. \\
& \left.\left.+\frac{z_{s} p \Delta x_{\min }}{R^{2}}\right)\right] \mathrm{d} x_{s} \mathrm{~d} z_{s},
\end{aligned}
$$

where the variable $\hat{x}$ is replaced by $p=\hat{x} \Delta x /$ $\Delta x_{\min }$. The source's intensity distribution is reconstructed from the 2-D inverse Fourier transform of the measured degree of coherence, i.e.,

$$
I_{s}\left(x_{s}, z_{s}\right) \propto \operatorname{IFT}_{2-\mathrm{D}}\left[\mu\left(\frac{\Delta x}{\lambda R}, \frac{p \Delta x_{\min }}{\lambda R^{2}}\right)\right],
$$

where IFT $_{2-\mathrm{D}}$ indicates a 2-D inverse Fourier transform. Equation (6) describes the reconstruction procedure of the source from the measured data.

The incoherent source in the following experiment is a two-point object produced by rotating a diffuser in front of two laser beams. Because of the different distance of each point source to the detection setup, the points are distributed in the $\left(x_{s}, z_{s}\right)$ plane (see Fig. 2). The changeable pinholes are made by two slits tilted toward each other. Along each line parallel to the $x$ axis there are effectively two pinholes with different distances between them. We change the pinholes' center of gravity by moving the slits together in parallel to the $x$ axis. For each slit's location $\hat{x}$, a set of interference gratings, each for a different $\Delta x$, is obtained in the back focal plane of the cylindrical lens. The experiment's parameters are $\lambda=0.63 \mu \mathrm{m}, R=$ $71 \mathrm{~cm}, \hat{x}_{\max }=9.5 \mathrm{~mm}, \Delta x=0.2-0.6 \mathrm{~mm}, \Delta z_{s}=9 \mathrm{~cm}$, and $\Delta x_{s}=\sim 2 \mathrm{~mm}$.

Sixty-four sets of interference gratings, five gratings in each, were measured. Each of the sets was obtained for a different pinhole's center of gravity $\hat{x}$. The visibility and the phase of each grating were measured and collected into the computer's memory. The complex visibility obtained from five gratings (five distances $\Delta x$ between the pinholes) in sixty-four different centers of gravity is shown in Figs. 3(a) and 3(b). Note that the frequency of the visibility function along $\hat{x}$ increases

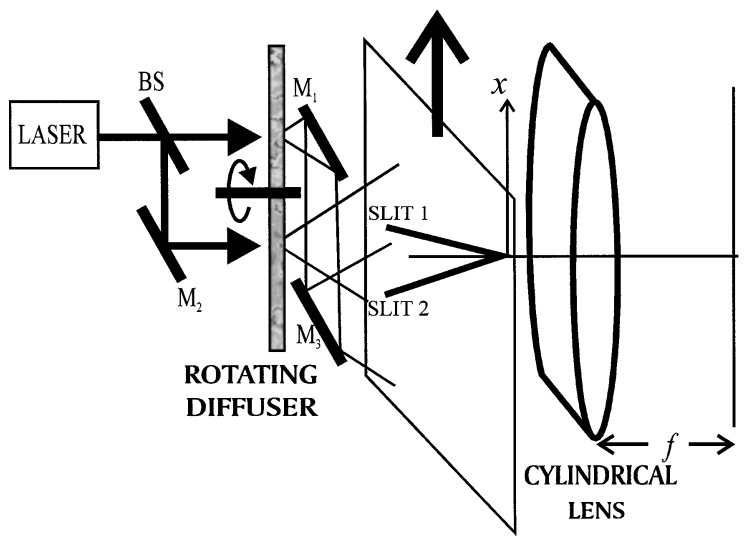

Fig. 2. Experimental setup: BS, beam splitter; M's, mirrors. 
(a)

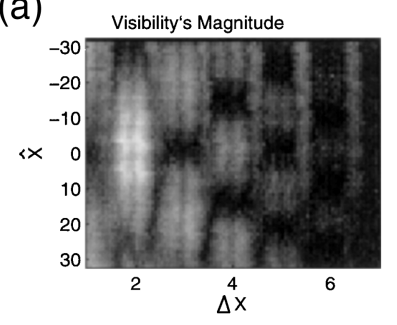

(b)

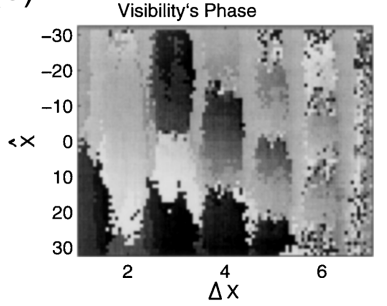

(c)

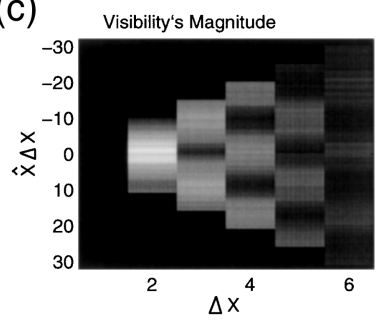

(d)

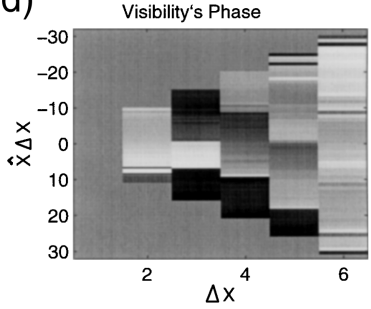

Fig. 3. (a) Magnitude and (b) phase of the degree of coherence as a function of $(\Delta x, \hat{x})$. (c), (d) Same as (a) and (b) after transformation of the coordinates to $\left(\Delta x, \hat{x} \Delta x / \Delta x_{\max }\right)$.

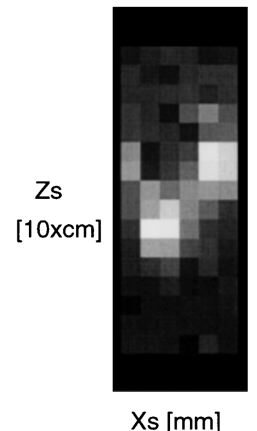

(a)

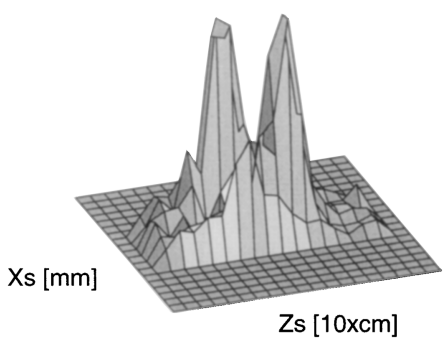

(b)

Fig. 4. Reconstruction of the source by an inverse Fourier transform of the complex function shown in Figs. 3(c) and 3(d). (a) Gray-level image, (b) 3-D plot.

linearly with $\Delta x$ and that the distance between any two peaks of brightness, in each $m$ th column [see Fig. 3(a)], follows the relation $\lambda R^{2} / \Delta z_{s} \Delta x_{m} \cong 3.53 \mathrm{~m}^{-1} \mathrm{~cm}, m=$ $2, \ldots 6$, as indeed expected from Eq. (5)

To reconstruct the source shape from the sampled complex visibility one first needs to transform the measured data to the coordinates $(\Delta x, p)$. To gain more longitudinal resolution it is worth changing the coordi-

nates $(\Delta x, \hat{x})$ to $\left(\Delta x, \hat{x} \Delta x / \Delta x_{\max }\right)$. The minimum object depth that can be resolved now is $\lambda R^{2} / \hat{x}_{\max } \Delta x_{\max }$ instead of $\lambda R^{2} / \hat{x}_{\max } \Delta x_{\min }$, as results from Eq. (5). As a result of this transformation the visibility plane is not completely occupied with measured data. Zero values are filled in the entire area of missing data, as is shown in Figs. 3(c) and 3(d).

The reconstruction of the source shape, shown in Fig. 4, was obtained by a 2-D inverse Fourier transformation of the complex visibility shown in Figs. 3(c) and 3(d). The zero padded areas in Figs. 3(c) and 3(d) can be considered a bandpass filtering and explain the noise that surrounds the two bright points in Fig. 4. One can extrapolate the visibility function into the zero padded areas and thus reduce the degradation in the reconstructed image. However, this subject is beyond the scope of the present preliminary demonstration.

In conclusion, a new kind of space-variant degree of coherence was experimentally demonstrated. This degree of coherence was obtained in the far field of an incoherent source distributed along the longitudinal axis. We demonstrated a reconstruction of an axially distributed source from this degree of coherence, measured by a Young-experiment setup. The experimental results confirm the theory developed in Refs. 2 and 3. Extending the system to three dimensions promises a new method of incoherent holography and 3-D imaging.

This research was supported by the U.S. Army Research Office and the Advanced Research Projects Agency.

*Present address, Department of Electrical and Computer Engineering, Ben-Gurion University of the Negev, P.O. Box 653, Beer-Sheva 84105, Israel.

\section{References}

1. J. T. Armstrong, D. J. Hutter, K. J. Johnston, and D. Mozurkewich, Phys. Today 48(5), 42 (1995).

2. J. Rosen and A. Yariv, J. Opt. Soc. Am. A 13, 2091 (1996).

3. J. Rosen and A. Yariv, Opt. Lett. 21, 1011 (1996).

4. L. Mandel and E. Wolf, Optical Coherence and Quantum Optics (Cambridge U. Press, Cambridge, 1995), Chap. 4, p. 150.

5. J. W. Goodman, Statistical Optics, 1st ed. (Wiley, New York, 1985), Chap. 5, p. 208.

6. M. Born and E. Wolf, Principles of Optics, 4th ed. (Pergamon, Oxford, 1970), Chap. 10, p. 509. 